\title{
Corneal Densitometry in Bilateral Keratoconus Patients with Unilateral Corneal Vogt's Striae: A Contralateral Eye Study
}

Shengsheng Wei

Tianjin Medical University

Jing Li

Shaanxi Eye Hospital, Xi'an People's Hospital, Xi'an Jiaotong University

Yong Li

Shaanxi Eye Hospital, Xi'an People's Hospital, Xi'an Jiaotong University

Yaohua Zhang

Shaanxi Eye Hospital, Xi'an People's Hospital, Xi'an Jiaotong University

Yan Cai

Shaanxi Eye Hospital, Xi'an People's Hospital, Xi'an Jiaotong University

Jing Du

Shaanxi Eye Hospital, Xi'an People's Hospital, Xi'an Jiaotong University

Jianguo Liu

Shaanxi Eye Hospital, Xi'an People's Hospital, Xi'an Jiaotong University

Yan Wang ( $\nabla$ wangyan7143@vip.sina.com )

Tianjin Medical University

Research Article

Keywords: Keratoconus, Cornea, Corneal densitometry, Vogt's striae, Contralateral eye study

Posted Date: August 2nd, 2021

DOI: https://doi.org/10.21203/rs.3.rs-729614/v1

License: @) (7) This work is licensed under a Creative Commons Attribution 4.0 International License. Read Full License 


\section{Abstract}

Background

We aimed to investigate corneal densitometry and correlations with corneal morphological parameters in bilateral keratoconus (KCN) patients with unilateral Vogt's striae.

Methods

One hundred and-twelve patients (224 eyes) with bilateral KCN and unilateral Vogt's striae were enrolled in this cross-sectional contralateral eye study. Corneal densitometry and morphological parameters were measured using Pentacam HR.

Results

Our study revealed that corneal densitometry values of the anterior $0-2 \mathrm{~mm}$ and 2-6 $\mathrm{mm}$, intermediate $0-2 \mathrm{~mm}$ and 2-6 $\mathrm{mm}$, posterior 2-6 mm, and total cornea 2-6 mm in eyes with Vogt's striae were significantly higher than those in eyes without Vogt's striae $(P<0.05)$. The corneal densitometry values of the anterior 6-10 mm, posterior 0-2 mm, and total cornea 6-10 mm were significantly lower in eyes with Vogt's striae $(P<0.05)$. The corneal densitometry values in the anterior 0-2 mm and total cornea 2-6 mm were positively correlated with $\mathrm{K} 1, \mathrm{~K} 2, \mathrm{Km}$, Kmax, ACE, and PCE $(P<0.05)$ and negatively correlated with CCT and TCT in eyes with Vogt's striae $(P<0.05) . \mathrm{K} 2, \mathrm{Km}$, and Kmax were significantly correlated with densitometry values of the anterior $0-2 \mathrm{~mm}$ and the intermediate $0-2 \mathrm{~mm}$ in eyes without Vogt's striae $(P<0.05)$. Corneal densitometry values of the anterior and intermediate layers in the central cornea were higher in KCN eyes with Vogt's striae than in eyes without Vogt's striae, and the densitometry values of the anterior corneal stroma in the central region of the cornea were higher than those of the peripheral cornea in $\mathrm{KCN}$ eyes with and without Vogt's striae.

Conclusions

In conclusion, these results indicate that Vogt's striae mainly occur on the anterior and intermediate layers during the progression of KCN.

\section{Background}

Keratoconus $(\mathrm{KCN})$ is a non-inflammatory and progressive corneal ectatic disorder characterized by central or paracentral thinning and steepening that produces a cone-shaped protrusion of the cornea and gradually impairs its optical features [1-3]. The incidence of KCN ranges from $0.05-2.5 \%$. In progressive KCN, stromal thinning, corneal scars, Fleischer's ring, and Vogt's striae can be seen on slit-lamp biomicroscopy [4]. Vogt's striae are vertical lines in the posterior stroma and Descemet's membrane that are parallel to the axis of the cone $[5,6]$. This is one of the typical clinical signs of $\mathrm{KCN}$ and plays an important role in KCN evaluation, classification, monitoring, and rehabilitation [7-12]. However, the cause of Vogt's striae is not clear. Mehmet et al. [13] evaluated alterations in corneal keratocytes, endothelial cell densities, stromal nerve thickness, and sub-basal nerve density in KCN patients using in vivo confocal microscopy (IVCM). The results demonstrated an association between the microstructural changes seen in KCN and Vogt's striae. To the best of our knowledge, the question of whether alterations of corneal densitometry are associated with the appearance of Vogt's striae has not been examined previously.

Corneal densitometry, also known as corneal backscattering, is considered an indicator of corneal transparency [14]. Corneal disarranged histology, including changes in corneal lamellar array and spacing, haze clouding, inflammation, and scarring formation, may compromise corneal transparency [15-17]. Previous studies have demonstrated that corneal densitometry values observed in KCN are statistically significantly different from that of a normal cornea [18, 19]. Many previous articles have assessed corneal tomography, biomechanics, and endothelial cell distributions in KCN eyes [20-22], but there have been few reports on changes in corneal densitometry in KCN patients with unilateral corneal Vogt's striae.

In this study, we evaluated corneal densitometry at different positions using the Pentacam HR (Pentacam HR, Oculus, Inc., Wetzlar, Germany) to gain detailed insight into corneal densitometry and to compare corneal densitometric properties in patients with bilateral KCN and unilateral Vogt's striae. We determined whether corneal densitometry values can effectively assess the severity of keratoconus and explored alterations observed in corneal collagen fiber in the progression of $\mathrm{KCN}$.

\section{Methods}

\section{Participants}

This prospective, contralateral eye study comprised 112 patients ( 224 eyes; 65 men and 47 women with a mean age of $23.93 \pm 6.81$ years). All patients presented at Xi'an People's Hospital(Xi'an Fourth Hospital) in Shaanxi Province (northwest China) with bilateral KCN and unilateral Vogt's striae between February 2018 and June 2020. Participants underwent a comprehensive ophthalmic examination by the same ophthalmologist. The examination included patient history, slit-lamp biomicroscopy, and tests of uncorrected visual acuity (UCVA), best corrected visual acuity (BCVA), intraocular pressure (Topcon CT-80, Topcon Corporation, Tokyo, Japan), axial length (A-SCAN PLUS, Accutome, Inc., Florida, America), cycloplegic refraction, and funduscopy. Scheimpflug-based anterior segment tomography (Pentacam HR, Oculus, Inc., Wetzlar, Germany) was applied to evaluate corneal densitometry values and corneal topographic parameters, including the flat keratometry value (K1), the steep keratometry value (K2), the mean keratometry value (Km), the maximum keratometry value (Kmax), central corneal thickness (CCT), the thinnest corneal thickness (TCT), anterior corneal elevation (ACE), and posterior corneal elevation (PCE).

Inclusion criteria for $\mathrm{KCN}$ eyes were increased curvature of the anterior surface of the corneal topography and that the point of the anterior and posterior surface elevation of the cornea was coincident with the thinnest point of the cornea upon examination with the Pentacam HR. Obvious clinical signs (i.e., 
Fleischer's ring, Vogt's striae, apical thinning) were seen under slit-lamp biomicroscopy [23]. All participants presented with bilateral keratoconus and unilateral corneal Vogt's striae. The exclusion criteria were viral keratitis, corneal opacities, ocular infection, history of corneal trauma, corneal refractive or intraocular surgery, and systemic diseases such as diabetes or connective tissue diseases.

The study protocol adhered to the tenets of the Declaration of Helsinki and received approval from the institutional review board and Ethics Committee of Xi'an People's Hospital(Xi'an Fourth Hospital). Written informed consent was obtained from all participants.

\section{Corneal Densitometry}

Corneal densitometry was assessed with a rotating Scheimpflug imaging system (Pentacam HR; Oculus, Inc., Wetzlar, Germany). During the examination, the patient's chin was placed on the chin rest and their forehead was placed against the forehead strap. The patient was asked to blink a few times and then open both eyes and stare at the fixation target. After attaining perfect alignment, the instrument automatically took 25 Scheimpflug images by rotating 360 degrees around the optical axis of the eye within $2 \mathrm{~s}$. Three consecutive measurements were performed on each eye. Only cases with acceptable quality images were included in the study. One experienced examiner conducted all the measurements.

Corneal densitometry values were measured in grayscale units (GSUs) based on a minimum light scattering of 0 (maximum transparency) and a maximum light scattering of 100 (minimum transparency). The cornea in the $12 \mathrm{~mm}$ area was divided into 4 concentric radiation areas for local densitometry analysis, including the central area (diameter: 0-2 mm), an annulus with an inner diameter of $2 \mathrm{~mm}$ and an outer diameter of $6 \mathrm{~mm}$ (referred to as the 2-6 mm annulus), a second annulus that was 6-10 $\mathrm{mm}$ in diameter (i.e., the 6-10 mm annulus). The outermost layer has a ring gap of 10-12 $\mathrm{mm}$. Corneal densitometry measurements were performed at different depths of the cornea: the anterior cornea (the first $120 \mu \mathrm{m}$ of the corneal thickness), the posterior cornea (the last $60 \mu \mathrm{m}$ of the corneal thickness), and the intermediate layer.

\section{Statistical analysis}

We present descriptive statistics as means and standard deviations. SPSS version 26.0 (SPSS, Chicago, IL, USA) was implemented for all statistical analyses. The normal distribution of parameters was assessed using the Kolmogorov-Smirnov test. Paired sample t-tests were used to compare parameters with a normal distribution and the Wilcoxon signed-rank test was used to compare non-parametric parameters. Pearson's correlation tests were used to examine correlations between scale values fitting a normal distribution. Spearman correlation tests were implemented to determine correlations between data with a skewed distribution as well as ranked ordinal data. Two-sided $P$ values less than 0.05 were considered statistically significant.

\section{Results}

We observed statistically significant differences in refractive outcomes between keratoconus eyes with and without Vogt's striae (all $P<0.001)$. As shown in Table 1, KCN eyes with Vogt's striae had statistically significantly higher absolute values of sphere, cylinder, and spherical equivalents compared to eyes with no Vogt's striae. The UCVA and BCVA in KCN eyes with Vogt's striae were statistically significantly lower than in eyes without Vogt's striae $(P<0.001)$.

Table 1

Contralateral comparison of basic parameters between KCN eyes with and without Vogt's striae

\begin{tabular}{|lllc|}
\hline Parameters & With Vogt's striae & Without Vogt's striae & t/S-value \\
\cline { 2 - 4 } & Mean \pm SD & Mean \pm SD & \\
\hline Sph(D) & $-7.25 \pm 4.31$ & $-4.86 \pm 2.64$ & $-5.332 \mathrm{a}$ \\
\hline Cyl(D) & $-4.28 \pm 2.77$ & $-2.52 \pm 2.04$ & $-5.495 \mathrm{a}$ \\
\hline SE(D) & $-9.39 \pm 4.48$ & $-6.11 \pm 3.1$ & $<0.001^{*}$ \\
UCVA(Log MAR) & $1.05 \pm 0.43$ & $0.77 \pm 0.41$ & $-6.867 \mathrm{a}$ \\
\hline BCVA(Log MAR) & $0.44 \pm 0.37$ & $0.12 \pm 0.16$ & $5.037 \mathrm{a}$ \\
\hline Abbrevition & $<0.001^{*}$ \\
\hline
\end{tabular}

Abbreviations: Sph: Sphere, Cyl: Cylinder, SE: Spherical equivalent, UDVA: Uncorrected Visual Acuity, BCVA: Best Corrected Visual Acuity, logMAR: Logarithm of the minimum angle of resolution

Notes: a-Paired-samples t-test. b-Wilcoxon signed ranks test. ${ }^{*} P$-value $<0.05$ is statistically significant

The main corneal morphological parameters are presented in Table 2. We found statistically significant differences in corneal morphological data (K1, K2, Km, Kmax, CCT, TCT, ACD, ACE, PCE) measured by the Pentacam HR between eyes with and without Vogt's striae (all $P<0.001)$. 
Table 2

Contralateral comparison of morphological parameters between $\mathrm{KCN}$ eyes with and without Vogt's striae

\begin{tabular}{|c|c|c|c|c|}
\hline \multirow[t]{2}{*}{ Parameters } & \multirow{2}{*}{$\begin{array}{l}\text { With Vogt's striae } \\
\text { Mean } \pm \text { SD }\end{array}$} & \multirow{2}{*}{$\begin{array}{l}\text { Without Vogt's striae } \\
\text { Mean } \pm \text { SD }\end{array}$} & \multirow[t]{2}{*}{ t/S-value } & \multirow[t]{2}{*}{$P$-value } \\
\hline & & & & \\
\hline K1(D) & $48.08 \pm 5.22$ & $43.82 \pm 1.81$ & $2506.5 b$ & $<0.001^{\star}$ \\
\hline K2(D) & $52.21 \pm 5.85$ & $46.12 \pm 2.85$ & $2649.5 b$ & $<0.001^{*}$ \\
\hline $\mathrm{Km}(\mathrm{D})$ & $50.04 \pm 5.40$ & $44.96 \pm 2.15$ & $2711.0 \mathrm{~b}$ & $<0.001^{\star}$ \\
\hline $\mathrm{Kmax}(\mathrm{D})$ & $59.18 \pm 9.38$ & $49.28 \pm 4.73$ & $2632.0 \mathrm{~b}$ & $<0.001^{\star}$ \\
\hline ССТ $(\mu \mathrm{m})$ & $450.56 \pm 35.39$ & $487.29 \pm 30.07$ & $-2685.0 b$ & $<0.001^{*}$ \\
\hline TCT $(\mu \mathrm{m})$ & $439.58 \pm 48.28$ & $479.33 \pm 30.03$ & $-2640.5 b$ & $<0.001^{*}$ \\
\hline $\operatorname{ACD}(\mu \mathrm{m})$ & $3.44 \pm 0.26$ & $3.34 \pm 0.26$ & $1966.5 b$ & $<0.001^{*}$ \\
\hline $\mathrm{ACE}(\mu \mathrm{m})$ & $33.43 \pm 18.23$ & $13.44 \pm 9.7$ & $2613.5 b$ & $<0.001^{\star}$ \\
\hline PCE $(\mu \mathrm{m})$ & $68.6 \pm 33.75$ & $33.06 \pm 19.02$ & $2592.5 b$ & $<0.001$ * \\
\hline
\end{tabular}

Abbreviations: K1: Flat Keratometry, K2: Steep Keratometry, Km: Mean Keratometry, Kmax: maximum keratometry, CCT: Central Corneal Thickness, TCT: Thinnest Corneal Thickness, ACD: Anterior Chamber Depth, ACE: Anterior Corneal Elevation, PCE: Posterior Central Elevation, D: Diopter; $\mu$ m, micron

Notes: a-Paired-samples t-test. b-Wilcoxon signed ranks test. ${ }^{*} P$-value $<0.05$ is statistically significant

\section{Corneal densitometry}

As shown in Table 3, we detected statistically significant differences in the distribution of densitometry values. Paired sample t-tests and Wilcoxon signed-rank tests revealed that the corneal densitometry values of the anterior 0-2 and 2-6 mm, the intermediate 0-2 and 2-6 mm, the posterior 2-6 mm, and the total cornea 2-6 mm in eyes with Vogt's striae were statistically significantly higher than in eyes with no Vogt's striae $(P<0.05)$. These values were statistically significantly lower for the corneal densitometry values of the anterior 6-10 mm, the posterior 0-2 mm, and the total cornea 6-10 mm in eyes with Vogt's striae $c(P<0.05)$. No statistically significant differences between the two groups were calculated in other locations $(P>0.05)$.

Table 3

Contralateral comparison of mean corneal densitometry values were measured by the Pentacam HR

\begin{tabular}{|lllll|}
\hline \multicolumn{5}{l}{ Letween KCN eyes with and without Vogt's striae } \\
& With Vogt's striae & Without Vogt's striae & \multirow{2}{*}{ t/S-value } & P-value \\
\cline { 2 - 4 } & Mean \pm SD & Mean \pm SD & & \\
\hline Anterior 0-2 & $22.76 \pm 3.01$ & $21.97 \pm 3.14$ & $1301.0 \mathrm{~b}$ & $<0.001^{*}$ \\
\hline Anterior 2-6 & $21.65 \pm 19.05$ & $19.34 \pm 2.56$ & $1354.5 \mathrm{~b}$ & $<0.001^{*}$ \\
\hline Anterior 6-10 & $18.09 \pm 3.64$ & $18.87 \pm 3.99$ & $-1428.5 \mathrm{~b}$ & $<0.001^{*}$ \\
\hline Anterior-total & $21.67 \pm 3.04$ & $22.03 \pm 3.33$ & $-551.5 \mathrm{~b}$ & 0.070 \\
\hline Intermediate 0-2 & $15.50 \pm 1.44$ & $15.19 \pm 1.59$ & $4.007 \mathrm{a}$ & $0.001^{*}$ \\
\hline Intermediate 2-6 & $13.39 \pm 1.12$ & $13.28 \pm 1.20$ & $732.0 \mathrm{~b}$ & $0.009 *$ \\
\hline Intermediate 6-10 & $12.98 \pm 2.13$ & $13.05 \pm 2.10$ & $-435.0 \mathrm{~b}$ & 0.129 \\
\hline Intermediate-total & $14.79 \pm 1.63$ & $14.89 \pm 1.73$ & $-0.806 \mathrm{a}$ & 0.422 \\
\hline Posterior 0-2 & $10.46 \pm 1.76$ & $11.17 \pm 1.38$ & $-1501.5 \mathrm{~b}$ & $<0.001^{*}$ \\
\hline Posterior 2-6 & $11.08 \pm 0.95$ & $10.72 \pm 1.04$ & $5.068 \mathrm{a}$ & $<0.001^{*}$ \\
\hline Posterior 6-10 & $11.11 \pm 1.53$ & $11.20 \pm 1.41$ & $-483.5 \mathrm{~b}$ & 0.107 \\
\hline Posterior-total & $11.77 \pm 1.18$ & $11.83 \pm 1.14$ & $-0.643 \mathrm{a}$ & 0.522 \\
\hline Total0-2 & $16.24 \pm 1.42$ & $16.11 \pm 1.54$ & $1.287 \mathrm{a}$ & 0.201 \\
\hline Total2-6 & $14.78 \pm 1.23$ & $14.45 \pm 1.29$ & $4.914 \mathrm{a}$ & $<0.001^{*}$ \\
\hline Total6-10 & $14.06 \pm 2.28$ & $14.38 \pm 2.36$ & $-1115.5 \mathrm{~b}$ & $<0.001^{*}$ \\
\hline Total & $16.08 \pm 1.62$ & $16.25 \pm 1.77$ & $-1.498 \mathrm{a}$ & 0.137 \\
\hline Notes: a-Pared & & & & \\
\hline
\end{tabular}

Notes: a-Paired-samples t-test. b-Wilcoxon signed ranks test. ${ }^{*} P$-value $<0.05$ is statistically significant 
Correlations between corneal densitometry and morphological parameters in eyes with Vogt's striae are shown in Table 4 and Figure 1. The corneal densitometry values in the anterior 0-2 mm and the total cornea 2-6 mm were positively correlated with $\mathrm{K} 1, \mathrm{~K} 2, \mathrm{Km}, \mathrm{Kmax}, \mathrm{ACE}$ and PCE ( $P<0.05)$ and negatively correlated with $\mathrm{CCT}$ and TCT $(P<0.05)$. The corneal densitometry values in the posterior $0-2 \mathrm{~mm}$ were negatively correlated with $\mathrm{K} 1$, $\mathrm{K} 2$, $\mathrm{Km}$, $\mathrm{Kmax}$, ACE and PCE $(P<0.05)$ and positively correlated with CCT $(P<0.05)$. The corneal densitometry values in the anterior 2-6 mm were positively correlated with $\mathrm{K} 1$, $\mathrm{K} 2, \mathrm{Km}, \mathrm{Kmax}, \mathrm{ACE}$, and PCE $(P<0.05)$ and negatively correlated with TCT $(P<0.05)$. We observed negative correlations between ACD and the anterior 6-10 mm, the anterior total, the intermediate $6-10 \mathrm{~mm}$, the intermediate total, the posterior $6-10 \mathrm{~mm}$, the posterior total, the total $6-10 \mathrm{~mm}$, and total values $(P<0.05)$.

Table 4

Correlation between corneal densitometry values and morphological parameters measured by the Pentacam HR in the eyes with Vo:

\begin{tabular}{|c|c|c|c|c|c|c|c|c|c|c|c|c|c|c|c|}
\hline \multirow[t]{2}{*}{ Location } & \multicolumn{2}{|l|}{ K1 } & \multicolumn{2}{|l|}{$\mathrm{K} 2$} & \multicolumn{2}{|l|}{$\mathrm{Km}$} & \multicolumn{2}{|l|}{ Kmax } & \multicolumn{2}{|l|}{ ССT } & \multicolumn{2}{|l|}{ TCT } & \multicolumn{2}{|l|}{ ACD } & \multirow{2}{*}{$\frac{A}{r}$} \\
\hline & $r$ & $P$ & $r$ & $P$ & $r$ & $P$ & $r$ & $P$ & $r$ & $P$ & $r$ & $P$ & $r$ & $P$ & \\
\hline Anterior 0-2 & 0.275 & $0.004^{*}$ & 0.329 & $0.000^{*}$ & 0.325 & $0.001 *$ & 0.289 & $0.002^{*}$ & -0.279 & $0.003^{*}$ & -0.211 & $0.028^{*}$ & -0.030 & 0.750 & 0. \\
\hline Anterior 2-6 & 0.213 & $0.026^{*}$ & 0.301 & $0.001^{*}$ & 0.278 & $0.004^{*}$ & 0.311 & $0.001^{*}$ & -0.196 & $0.041^{*}$ & -0.143 & 0.139 & 0.002 & 0.979 & 0. \\
\hline $\begin{array}{l}\text { Anterior 6- } \\
10\end{array}$ & -0.103 & 0.286 & -0.018 & 0.846 & -0.057 & 0.558 & -0.018 & 0.846 & -0.023 & 0.808 & -0.015 & 0.873 & -0.373 & $0.000^{*}$ & -0 \\
\hline Anterior-total & -0.048 & 0.620 & 0.030 & 0.754 & 0.003 & 0.974 & 0.046 & 0.630 & -0.043 & 0.652 & -0.011 & 0.909 & -0.278 & $0.003^{*}$ & -0 \\
\hline $\begin{array}{l}\text { Intermediate } \\
0-2\end{array}$ & 0.151 & 0.117 & 0.162 & 0.093 & 0.174 & 0.071 & 0.115 & 0.235 & -0.253 & $0.008^{*}$ & -0.271 & $0.004^{*}$ & -0.040 & 0.679 & 0. \\
\hline $\begin{array}{l}\text { Intermediate } \\
2-6\end{array}$ & -0.016 & 0.867 & 0.022 & 0.819 & 0.003 & 0.972 & 0.011 & 0.908 & -0.100 & 0.300 & -0.164 & 0.090 & -0.058 & 0.545 & 0. \\
\hline $\begin{array}{l}\text { Intermediate } \\
6-10\end{array}$ & -0.087 & 0.366 & -0.031 & 0.749 & -0.063 & 0.515 & -0.064 & 0.506 & -0.026 & 0.787 & -0.080 & 0.411 & -0.406 & $0.000^{*}$ & -0 \\
\hline $\begin{array}{l}\text { Intermediate- } \\
\text { total }\end{array}$ & -0.118 & 0.220 & -0.096 & 0.320 & -0.109 & 0.257 & -0.114 & 0.237 & -0.013 & 0.890 & -0.077 & 0.426 & -0.352 & $0.000^{*}$ & -0 \\
\hline $\begin{array}{l}\text { Posterior 0- } \\
2\end{array}$ & -0.357 & $0.000 *$ & -0.357 & $0.000^{*}$ & -0.367 & $0.000 *$ & -0.344 & $0.000 *$ & 0.203 & $0.034^{*}$ & 0.114 & 0.240 & -0.062 & 0.522 & -0 \\
\hline $\begin{array}{l}\text { Posterior 2- } \\
6\end{array}$ & 0.131 & 0.175 & 0.179 & 0.062 & 0.165 & 0.087 & 0.252 & $0.008^{*}$ & -0.137 & 0.157 & -0.186 & 0.054 & -0.014 & 0.878 & 0. \\
\hline $\begin{array}{l}\text { Posterior 6- } \\
10\end{array}$ & -0.086 & 0.373 & -0.041 & 0.668 & -0.067 & 0.491 & -0.033 & 0.727 & -0.064 & 0.508 & -0.113 & 0.242 & -0.374 & $0.000^{*}$ & -0 \\
\hline $\begin{array}{l}\text { Posterior- } \\
\text { total }\end{array}$ & -0.132 & 0.173 & -0.113 & 0.242 & -0.126 & 0.190 & -0.061 & 0.527 & -0.036 & 0.708 & -0.097 & 0.318 & -0.308 & $0.001^{*}$ & -0 \\
\hline Total0-2 & 0.131 & 0.176 & 0.171 & 0.076 & 0.170 & 0.078 & 0.135 & 0.160 & -0.224 & $0.019 *$ & -0.221 & $0.021^{*}$ & -0.054 & 0.577 & 0. \\
\hline Total2-6 & 0.199 & $0.038^{*}$ & 0.296 & $0.001^{\star}$ & 0.264 & $0.005^{\star}$ & 0.333 & $0.000 *$ & -0.236 & $0.013^{*}$ & -0.226 & $0.018^{*}$ & -0.004 & 0.963 & 0. \\
\hline Total6-10 & -0.120 & 0.213 & -0.040 & 0.678 & -0.083 & 0.391 & -0.049 & 0.610 & -0.018 & 0.849 & -0.044 & 0.652 & -0.416 & $0.000 *$ & -0 \\
\hline Total & -0.117 & 0.225 & -0.044 & 0.649 & -0.076 & 0.433 & -0.036 & 0.708 & -0.032 & 0.742 & -0.039 & 0.688 & -0.385 & $0.000 *$ & -0 \\
\hline
\end{tabular}

Abbreviations: K1: Flat Keratometry, K2: Steep Keratometry, Km: Mean Keratometry, Kmax: maximum keratometry, CCT: Central Corneal Thickness, TCT: Thin Anterior Chamber Depth, ACE: Anterior Corneal Elevation, PCE: Posterior Central Elevation

Notes: Spearman correlation coefficient analysis. ${ }^{*} P$-value $<0.05$ is statistically significant

\section{Corneal densitometry and morphological parameters}

As presented in Table 5, there was no correlation between $\mathrm{K} 1$ and corneal densitometry values at any location in KCN eyes without Vogt's striae ( $P>0.05)$. K2, $\mathrm{Km}$, and $\mathrm{Kmax}$ were significantly correlated with the densitometry values of the anterior $0-2 \mathrm{~mm}$ and the intermediate $0-2 \mathrm{~mm}(P<0.05)$, but not with the posterior 0-2 $\mathrm{mm}$ ( $P>0.05)$. The corneal densitometry values in the intermediate 0-2 $\mathrm{mm}$ and 2-6 mm were negatively correlated with CCT and TCT ( $P<0.05)$ and positively correlated with ACE and PCE in KCN eyes without Vogt's striae $(P<0.05)$. We observed negative correlations between ACD and the anterior 6-10 $\mathrm{mm}$, the anterior total, the intermediate $6-10 \mathrm{~mm}$, the intermediate total, the posterior $6-10 \mathrm{~mm}$, the posterior total, the total 6-10 mm, and total corneal densitometry values in $\mathrm{KCN}$ eyes without Vogt's striae $(P<0.05)$. 
Table 5

Correlation between corneal densitometry values and morphological parameters measured by the Pentacam HR in the eyes without $\mathrm{V}$

\begin{tabular}{|c|c|c|c|c|c|c|c|c|c|c|c|c|c|c|c|}
\hline \multirow[t]{2}{*}{ Location } & \multicolumn{2}{|l|}{ K1 } & \multicolumn{2}{|l|}{$\mathrm{K} 2$} & \multicolumn{2}{|l|}{$\mathrm{Km}$} & \multicolumn{2}{|l|}{$\mathrm{Kmax}$} & \multicolumn{2}{|l|}{ CCT } & \multicolumn{2}{|l|}{ TCT } & \multicolumn{2}{|l|}{ ACD } & \multirow{2}{*}{$\frac{\mathrm{AC}}{r}$} \\
\hline & $r$ & $P$ & $r$ & $P$ & $r$ & $P$ & $r$ & $P$ & $r$ & $P$ & $r$ & $P$ & $r$ & $P$ & \\
\hline Anterior 0-2 & 0.100 & 0.302 & 0.257 & $0.007 *$ & 0.200 & $0.029 *$ & 0.241 & $0.011^{*}$ & -0.021 & 0.831 & -0.020 & 0.833 & -0.075 & 0.634 & 0.1 \\
\hline Anterior 2-6 & 0.004 & 0.968 & 0.141 & 0.144 & 0.080 & 0.344 & 0.139 & 0.153 & -0.006 & 0.955 & 0.001 & 0.994 & -0.071 & 0.673 & $0 . C$ \\
\hline $\begin{array}{l}\text { Anterior 6- } \\
10\end{array}$ & -0.059 & 0.545 & -0.050 & 0.605 & -0.059 & 0.547 & -0.123 & 0.204 & 0.031 & 0.753 & 0.033 & 0.736 & -0.340 & $0.000 *$ & -0 \\
\hline Anterior-total & -0.050 & 0.610 & -0.043 & 0.658 & -0.058 & 0.715 & -0.073 & 0.451 & 0.031 & 0.753 & 0.040 & 0.678 & -0.263 & $0.005^{\star}$ & -0 . \\
\hline $\begin{array}{l}\text { Intermediate } \\
0-2\end{array}$ & 0.185 & 0.054 & 0.265 & $0.005^{\star}$ & 0.252 & ${ }_{*}^{0.006}$ & 0.264 & $0.005^{\star}$ & -0.271 & $0.004^{*}$ & -0.285 & $0.002^{*}$ & -0.086 & 0.608 & 0.2 \\
\hline $\begin{array}{l}\text { Intermediate } \\
2-6\end{array}$ & 0.126 & 0.195 & 0.160 & 0.099 & 0.155 & 0.082 & 0.168 & 0.082 & -0.236 & $0.013^{*}$ & -0.247 & $0.010 *$ & -0.061 & 0.875 & 0.2 \\
\hline $\begin{array}{l}\text { Intermediate } \\
6-10\end{array}$ & 0.097 & 0.318 & 0.054 & 0.578 & 0.079 & 0.445 & -0.038 & 0.694 & -0.093 & 0.339 & -0.094 & 0.338 & -0.326 & $0.000 *$ & -0 . \\
\hline $\begin{array}{l}\text { Intermediate- } \\
\text { total }\end{array}$ & 0.180 & 0.063 & 0.068 & 0.486 & 0.119 & 0.163 & 0.002 & 0.984 & -0.109 & 0.262 & -0.122 & 0.210 & -0.303 & $0.000 *$ & -0. \\
\hline $\begin{array}{l}\text { Posterior 0- } \\
2\end{array}$ & -0.041 & 0.673 & -0.086 & 0.374 & -0.065 & 0.647 & -0.122 & 0.208 & -0.003 & 0.974 & -0.026 & 0.792 & 0.050 & 0.470 & -0. \\
\hline $\begin{array}{l}\text { Posterior 2- } \\
6\end{array}$ & 0.072 & 0.460 & 0.137 & 0.156 & 0.108 & 0.203 & 0.144 & 0.135 & -0.078 & 0.421 & -0.066 & 0.496 & 0.085 & 0.207 & 0.2 \\
\hline $\begin{array}{l}\text { Posterior 6- } \\
10\end{array}$ & 0.073 & 0.455 & 0.012 & 0.898 & 0.036 & 0.674 & -0.086 & 0.379 & -0.053 & 0.583 & -0.041 & 0.676 & -0.301 & $0.000 *$ & -0 . \\
\hline $\begin{array}{l}\text { Posterior- } \\
\text { total }\end{array}$ & 0.105 & 0.279 & -0.008 & 0.931 & 0.037 & 0.595 & -0.070 & 0.468 & -0.042 & 0.667 & -0.044 & 0.651 & -0.234 & $0.005^{\star}$ & -0. \\
\hline Total0-2 & 0.114 & 0.241 & 0.222 & $0.021^{*}$ & 0.188 & $0.034^{*}$ & 0.214 & $0.025^{\star}$ & -0.112 & 0.247 & -0.126 & 0.193 & -0.102 & 0.501 & 0.1 \\
\hline Total2-6 & 0.049 & 0.614 & 0.155 & 0.109 & 0.108 & 0.208 & 0.163 & 0.092 & -0.096 & 0.324 & -0.099 & 0.310 & -0.067 & 0.780 & 0.1 \\
\hline Total6-10 & 0.011 & 0.907 & -0.010 & 0.916 & -0.003 & 0.978 & -0.104 & 0.286 & -0.001 & 0.989 & 0.002 & 0.986 & -0.353 & $0.000^{*}$ & -0 \\
\hline Total & 0.067 & 0.490 & -0.001 & 0.994 & 0.021 & 0.653 & -0.062 & 0.521 & 0.006 & 0.953 & 0.006 & 0.950 & -0.326 & $0.000 *$ & -0 . \\
\hline
\end{tabular}

Abbreviations: K1: Flat Keratometry, K2: Steep Keratometry, Km: Mean Keratometry, Kmax: maximum keratometry, CCT: Central Corneal Thickness, TCT: Thin Anterior Chamber Depth, ACE: Anterior Corneal Elevation, PCE: Posterior Central Elevation

Notes: Spearman correlation coefficient analysis. ${ }^{*} P$-value $<0.05$ is statistically significant

\section{Discussion}

$\mathrm{KCN}$ eyes were reported to have different cornea-related parameters compared with normal eyes, including corneal curvature, corneal thickness, corneal endothelial density, corneal hysteresis, and corneal resistance factor [8, 24-25]. Clinical manifestations and signs of keratoconus are different at different stages and mild keratoconus is usually difficult to detect with slit-lamp biomicroscopy. Fleischer's ring and Vogt's striae can be seen on the corneal inferior or center as the disease progresses. Vogt's striae or stress lines are typical clinical signs of progressive KCN [11]. We observed that diopter, visual acuity, and corneal characteristics were statistically significantly altered when Vogt's striae appeared. However, associations corneal morphological parameters, mechanisms of the formation of Vogt's striae, and where the striae first appeared are still ambiguous.

Our study found that KCN eyes with Vogt's striae had statistically higher absolute values of sphere, cylinder, and spherical equivalents compared to eyes without Vogt's striae, and the UCVA and BCVA in KCN eyes with Vogt's striae were statistically significantly lower than in eyes without Vogt's striae. This is consistent with previous research [26]. Sedaghat et al. [23] reported that morphological parameters (K1, K2, Kmax) measured by Pentacam HR in KCN eyes with Vogt's striae were higher than in eyes without Vogt's striae. In the present study, we evaluated additional morphological parameters (i.e., CCT, TCT, ACD, $\mathrm{ACE}$ and $\mathrm{PCE}$, in addition to the standard parameters of $\mathrm{K} 1, \mathrm{~K} 2, \mathrm{Km}$, and $\mathrm{Kmax}$ ). In addition to an increase in the anterior corneal curvature, results indicated statistically significant increases in ACE, PCE, and ACD, and statistically significant decreases in CCT and TCT in eyes with Vogt's striae. Thus, we can conclude that the occurrence of Vogt's striae is positively correlated with the severity of KCN. In the absence of corneal topography, keratoconus severity can be assessed by observing the presence of Vogt's striae under slit-lamp biomicroscopy during clinical examination.

Vogt's striae may affect the densitometry of the cornea as well as morphological parameters. To our knowledge, few studies have investigated associations between corneal densitometry and Vogt's striae in KCN eyes. As demonstrated in a previous study, several factors (i.e., the size and arrangement of collagen fibrils) could influence corneal transparency and densitometry [27]. The disarrangement of the corneal collagen structure in KCN patients [17] may lead to differences in corneal densitometry compared with normal subjects [28, 29]. 
In the current study, we found that corneal densitometry values of the anterior 0-2 and 2-6 $\mathrm{mm}$ and the intermediate 0-2 and 2-6 mm were statistically significantly higher, and that the posterior $0-2 \mathrm{~mm}$ was statistically significantly lower in KCN eyes with Vogt's striae (contrasted with KCN eyes without Vogt's striae). This is similar to the results observed by Shen et al [30] in a study on corneal densitometry in KCN. Alternations in corneal densitometry are related to the degree of changes in the structure of corneal collagen fiber [31]. In previous studies, Uçakhan et al. [32] and Ghosh et al. [33] reported that the presence of Vogt's striae in the anterior stroma of KCN eyes is usually present in moderate and severe KCN. Erie et al. [34] and Hollingsworth et al. [5] reported a decrease in keratocyte density, with a $19 \%$ reduction in the anterior stroma and a $10 \%$ reduction in the posterior stroma. We hypothesize that KCN eyes with Vogt's striae present with less epithelial cell density, less stromal keratocyte density in the anterior and intermediate stroma, greater stromal degeneration, and fewer intact corneal collagen fibrils. The abnormal separation of collagen fibrils and wound healing reactions in stromal lamellae may lead to increased light scattering of corneal tissue [35]. Hence, the corneal densitometry values of the anterior and intermediate corneal stroma are higher than that of the posterior stroma in KCN eyes with Vogt's striae (Fig. 2). Since the cone of KCN is mainly located in the central area as well as under the temporal area of the cornea [36], corneal densitometry changes within the $0-6 \mathrm{~mm}$ area of the cornea are more obvious.

As demonstrated in previous studies [22, 23, 26, 37], the UCVA and BCVA in KCN eyes with Vogt's striae were statistically significantly lower than in eyes without Vogt's striae, and corneal morphological parameters are clearly affected in KCN eyes with Vogt's striae. These results are consistent with our findings. We evaluated possible correlations between corneal densitometry results and corneal morphological parameters in KCN eyes with and without Vogt's striae. In $\mathrm{KCN}$ eyes with Vogt's striae, we found that corneal densitometry values in the anterior 0-2 and 2-6 mm and the total cornea (2-6 mm) were positively correlated with $\mathrm{K} 1, \mathrm{~K} 2, \mathrm{Km}, \mathrm{Kmax}, \mathrm{ACE}$ and PCE and negatively correlated with CCT and TCT. The severity of KCN is positively correlated with anterior surface curvature and the elevation of anterior and posterior surfaces and is negatively correlated with corneal thickness [38]. Therefore, corneal densitometry values within $0-6 \mathrm{~mm}$ of the anterior corneal layer can reflect the severity of $\mathrm{KCN}$. We speculate that misalignment of corneal collagen and the degeneration of fibronectin in the extracellular matrix occur in the anterior corneal stroma when Vogt's striae appear in KCN eyes. Corneal densitometry values in the central area of the cornea can be used to evaluate the efficacy and impact of corneal collagen cross-linking surgery for KCN [39].

In KCN eyes without Vogt's striae, we found that only K2, Km, and Kmax correlated with the densitometry values of the anterior $0-2 \mathrm{~mm}$ and intermediate $0-2$ $\mathrm{mm}$. There was no correlation between $\mathrm{K} 1$ and corneal densitometry values at any location. Since the orientation of most stromal bands usually occurs in the steepest Sim K axis of the cornea [37], the corneal ultrastructure of the flat corneal axis is less altered in eyes with mild KCN. We hypothesize that corneal densitometry in the central area may change in the early stages of $\mathrm{KCN}$. Therefore, in the evaluation and diagnosis of early $\mathrm{KCN}$, corneal densitometry in the central region can be used as an auxiliary diagnostic indicator.

We were not able to find a correlation between central corneal densitometry and ACD in KCN eyes with and without Vogt's striae. However, we found negative correlations between ACD and peripheral corneal densitometry in all patients. We postulate that those alternations in ACD may be associated with changes in corneal morphology, as described by other authors [2].

One limitation of our study is that participants were not grouped by corneal curvature; this may lead to potential bias. However, the main objective of the current study is to investigate changes in corneal densitometry in $\mathrm{KCN}$ eyes with Vogt's striae and to explore regions of alternations of corneal collagen fiber that occur with the progression of KCN. Therefore, curvature has little effect on our study. Another limitation of our study is that the examination of corneal densitometry in our study was not performed concurrently. We will thus more rigidly control inspection time to reduce errors in future investigations.

\section{Conclusion}

This study demonstrated that the corneal densitometry values of the anterior and intermediate layers in the central corneal were higher in KCN eyes with Vogt's striae than in KCN eyes without Vogt's striae, and that the densitometry of the anterior corneal stroma in the central region of the cornea was higher than that of the peripheral cornea in KCN eyes with and without Vogt's striae. Central corneal densitometry has a clear correlation with K1, K2, Km, Kmax, CCT, TCT, ACD, $\mathrm{ACE}$, and PCE in KCN eyes with Vogt's striae. These results demonstrate the association of corneal densitometry in the central anterior area with $\mathrm{KCN}$ severity. The results also indirectly indicate that collagen fibers and structures in the central anterior cornea were changed during the progression of $\mathrm{KCN}$, which provides important evidence for controlling the progress of KCN effectively through anterior corneal collagen cross-linking surgery.

\section{Abbreviations}

\section{Keratoconus: KCN}

In vivo confocal microscopy: IVCM

Uncorrected visual acuity: UCVA

Best corrected visual acuity: BCVA

Keratometry value: $\mathrm{K} 1$

Steep keratometry value: K2

Mean keratometry value: $\mathrm{Km}$

Maximum keratometry value: Kmax

Central corneal thickness: CCT 
Thinnest corneal thickness: TCT

Anterior corneal elevation: ACE

Posterior corneal elevation: PCE

\section{Declarations}

Ethics approval and consent to participate[This study protocol adhered to the tenets of the Declaration of Helsinki and received approval[A1] from the institutional review board and Ethics Committee of Xi'an People's Hospital(Xi'an Fourth Hospital). Written informed consent was obtained from all participants.

Consent for publication $\square$ Not applicable.

Availability of data and materials: The datasets used and analyzed during the current study are available from the corresponding author on reasonable request.

Competing interests: The authors declare that there is no conflict of interest regarding the publication of this paper.

Funding: This work was supported by the National Natural Science Foundation of China (No. 81873684).

Authors' contributions $\square$ Concept and design: Shengsheng Wei, Jing Li, Yan Wang. Data collection: Shengsheng Wei, Yan Cai, Jing Du. Analysis and interpretation: Shengsheng Wei, Yong Li, Jianguo Liu. Writing the article: Shengsheng Wei, Jing Li, Yaohua Zhang. Critical revision: Yan Wang. Final approval: all authors.

Acknowledgments: The authors are grateful for the technical support of Tianjin Eye Hospital \& Eye Institute (the Tianjin Key Lab of Ophthalmology and Visual Science).

Please include the approval number if applicable.

\section{References}

1. Blackburn BJ, Jenkins MW, Rollins AM, Dupps WJ. A Review of Structural and Biomechanical Changes in the Cornea in Aging, Disease, and Photochemical Crosslinking. Front Bioeng Biotechnol. 2019;7:66.

2. Romero-Jiménez M, Santodomingo-Rubido J, Wolffsohn JS. Keratoconus: a review. Cont Lens Anterior Eye. 2010;33(4):157-66.

3. Millodot M, Ortenberg I, Lahav-Yacouel K, Behrman S. Effect of ageing on keratoconic corneas. J Optom. 2016;9(2):72-7.

4. Zadnik K, Barr JT, Edrington TB, Everett DF, Jameson M, McMahon TT, et al. Baseline findings in the Collaborative Longitudinal Evaluation of Keratoconus (CLEK) Study. Invest Ophthalmol Vis Sci. 1998;39(13):2537-46.

5. Hollingsworth JG, Efron N. Observations of banding patterns (Vogt striae) in keratoconus: a confocal microscopy study. Cornea. 2005;24(2):162-6.

6. Güngör IU, Beden U, Sönmez B. Bilateral horizontal Vogt's striae in keratoconus. Clin Ophthalmol. 2008;2(3):653-5.

7. Rabinowitz YS. Keratoconus. Surv Ophthalmol. 1998;42(4):297-319.

8. Mas Tur V, MacGregor C, Jayaswal R, O'Brart D, Maycock N. A review of keratoconus: Diagnosis, pathophysiology, and genetics. Surv Ophthalmol. 2017;62(6):770-783.[A1]

9. Kennedy RH, Bourne WM, Dyer JA. A 48-year clinical and epidemiologic study of keratoconus. Am J Ophthalmol. 1986;101(3):267-73.

10. Gokhale NS. Epidemiology of keratoconus. Indian J Ophthalmol. 2013;61(8):382-3.

11. Grieve K, Ghoubay D, Georgeon C, Latour G, Nahas A, Plamann K, et al. Stromal striae: a new insight into corneal physiology and mechanics. Sci Rep. 2017;7(1):13584.

12. Wisse RPL, Simons RWP, van der Vossen MJB, Muijzer MB, Soeters N, Nuijts RMMA, et al. Clinical Evaluation and Validation of the Dutch Crosslinking for Keratoconus Score. JAMA Ophthalmol. 2019;137(6):610-616.

13. Mocan MC, Yilmaz PT, Irkec M, Orhan M. The significance of Vogt's striae in keratoconus as evaluated by in vivo confocal microscopy. Clin Exp Ophthalmol. 2008;36(4):329-34

14. Kreps EO, Jimenez-Garcia M, Issarti I, Claerhout I, Koppen C, Rozema JJ. Repeatability of the Pentacam HR in Various Grades of Keratoconus. Am J Ophthalmol. 2020;219:154-162.

15. Lopes B, Ramos I, Ambrósio R Jr. Corneal densitometry in keratoconus. Cornea. 2014;33(12):1282-6.

16. Ní Dhubhghaill S, Rozema JJ, Jongenelen S, Ruiz Hidalgo I, Zakaria N, Tassignon MJ. Normative values for corneal densitometry analysis by Scheimpflug optical assessment. Invest Ophthalmol Vis Sci. 2014;55(1):162-8.

17. Shen Y, Jian W, Sun L, Li M, Han T, Son J, et al. One-Year Follow-Up of Changes in Corneal Densitometry After Accelerated (45 mW/cm2) Transepithelial Corneal Collagen Cross-Linking for Keratoconus: A Retrospective Study. Cornea. 2016;35(11):1434-1440.

18. Bitirgen G, Ozkagnici A, Bozkurt B, Malik RA. In vivo corneal confocal microscopic analysis in patients with keratoconus. Int J Ophthalmol. 2015;8(3):5349. 
19. Ozgurhan EB, Kara N, Yildirim A, Bozkurt E, Uslu H, Demirok A. Evaluation of corneal microstructure in keratoconus: a confocal microscopy study. Am J Ophthalmol. 2013;156(5):885-893.e2.

20. Goebels S, Eppig T, Seitz B, Szentmàry N, Cayless A, Langenbucher A. Endothelial alterations in 712 keratoconus patients. Acta Ophthalmol. 2018;96(2):e134-e139.

21. El-Agha MS, El Sayed YM, Harhara RM, Essam HM. Correlation of corneal endothelial changes with different stages of keratoconus. Cornea. 2014;33(7):707-11.

22. Askarizadeh F, Sedaghat MR, Ostadi-Moghaddam H, Narooie-Noori F, Rakhshandadi T, Rajabi S. A Contralateral Eye Study Comparing Corneal Biomechanics in Subjects with Bilateral Keratoconus with Unilateral Vogt's Striae. Med Hypothesis Discov Innov Ophthalmol. 2017;6(2):49-55.

23. Sedaghat MR, Askarizadeh F, Narooie-Noori F, Rakhshandadi T, Ostadi-Moghadam H, Rajabi S. Comparative evaluation of tomographic and biometric characteristics in bilateral keratoconus patients with unilateral corneal Vogt's striae: a contralateral eye study. Clin Ophthalmol. 2018;12:1383-1390.

24. Mukhtar S, Ambati BK. Pediatric keratoconus: a review of the literature. Int Ophthalmol. 2018;38(5):2257-2266[A2] .

25. Steinberg J, Siebert M, Katz T, Frings A, Mehlan J, Druchkiv V, et al. Tomographic and Biomechanical Scheimpflug Imaging for Keratoconus Characterization: A Validation of Current Indices. J Refract Surg. 2018;34(12):840-847.

26. Rakhshandadi T, Sedaghat MR, Askarizadeh F, Momeni-Moghaddam H, Khabazkhoob M, Yekta A, et al. Refractive characteristics of keratoconus eyes with corneal Vogt's striae: A contralateral eye study. J Optom. 2021;14(2):183-188.

27. Freegard TJ. The physical basis of transparency of the normal cornea. Eye (Lond). 1997;11:465-71.

28. Rozema JJ, Koppen C, Bral N, Tassignon MJ. Changes in Forward and Backward Light Scatter in Keratoconus Resulting From Corneal Cross-Linking. Asia Pac J Ophthalmol (Phila). 2013;2(1):15-9.

29. Alnawaiseh M, Rosentreter A, Eveslage M, Eter N, Zumhagen L. Changes in Corneal Transparency After Cross-linking for Progressive Keratoconus: Longterm Follow-up. J Refract Surg. 2015;31(9):614-8.

30. Shen Y, Han T, Jhanji V, Shang J, Zhao J, Li M, et al. Correlation Between Corneal Topographic, Densitometry, and Biomechanical Parameters in Keratoconus Eyes. Transl Vis Sci Technol. 2019;8(3):12.

31. Chung SH, Kim EK. Keratoconus with unilateral horizontal stress lines. Cornea. 2005;24(7):890.

32. Uçakhan OO, Kanpolat A, YImaz N, Ozkan M. In vivo confocal microscopy findings in keratoconus. Eye Contact Lens. 2006;32(4):183-91.

33. Ghosh S, Mutalib HA, Kaur S, Ghoshal R, Retnasabapathy S. Corneal Cell Morphology in Keratoconus: A Confocal Microscopic Observation. Malays J Med Sci. 2017;24(2):44-54.

34. Erie JC, Patel SV, McLaren JW, Nau CB, Hodge DO, Bourne WM. Keratocyte density in keratoconus. A confocal microscopy study(a). Am J Ophthalmol. 2002;134(5):689-95.

35. Krachmer JH, Feder RS, Belin MW. Keratoconus and related noninflammatory corneal thinning disorders. Surv Ophthalmol. 1984;28(4):293-322.

36. Huseynli S, Salgado-Borges J, Alio JL. Comparative evaluation of Scheimpflug tomography parameters between thin non-keratoconic, subclinical keratoconic, and mild keratoconic corneas. Eur J Ophthalmol. 2018;28(5):521-534.

37. Ostadi-Moghaddam H, Sedaghat MR, Rakhshandadi T, Rajabi S, Narooie-Noori F, Askarizadeh F. A contralateral eye study comparing characteristics of corneal endothelial cells in bilateral keratoconus patients with unilateral corneal Vogt's striae. J Curr Ophthalmol. 2018;30(3):228-233.

38. Bühren J. Hornhauttopografie und Keratokonusdiagnostik mittels Scheimpflug-Fotografie [Corneal topography and keratoconus diagnostics with Scheimpflug photography]. Ophthalmologe. 2014;111(10):920-6. German.

39. Böhm M, Shajari M, Remy M, Kohnen T. Corneal densitometry after accelerated corneal collagen cross-linking in progressive keratoconus. Int Ophthalmol. 2019;39(4):765-775.

\section{Figures}



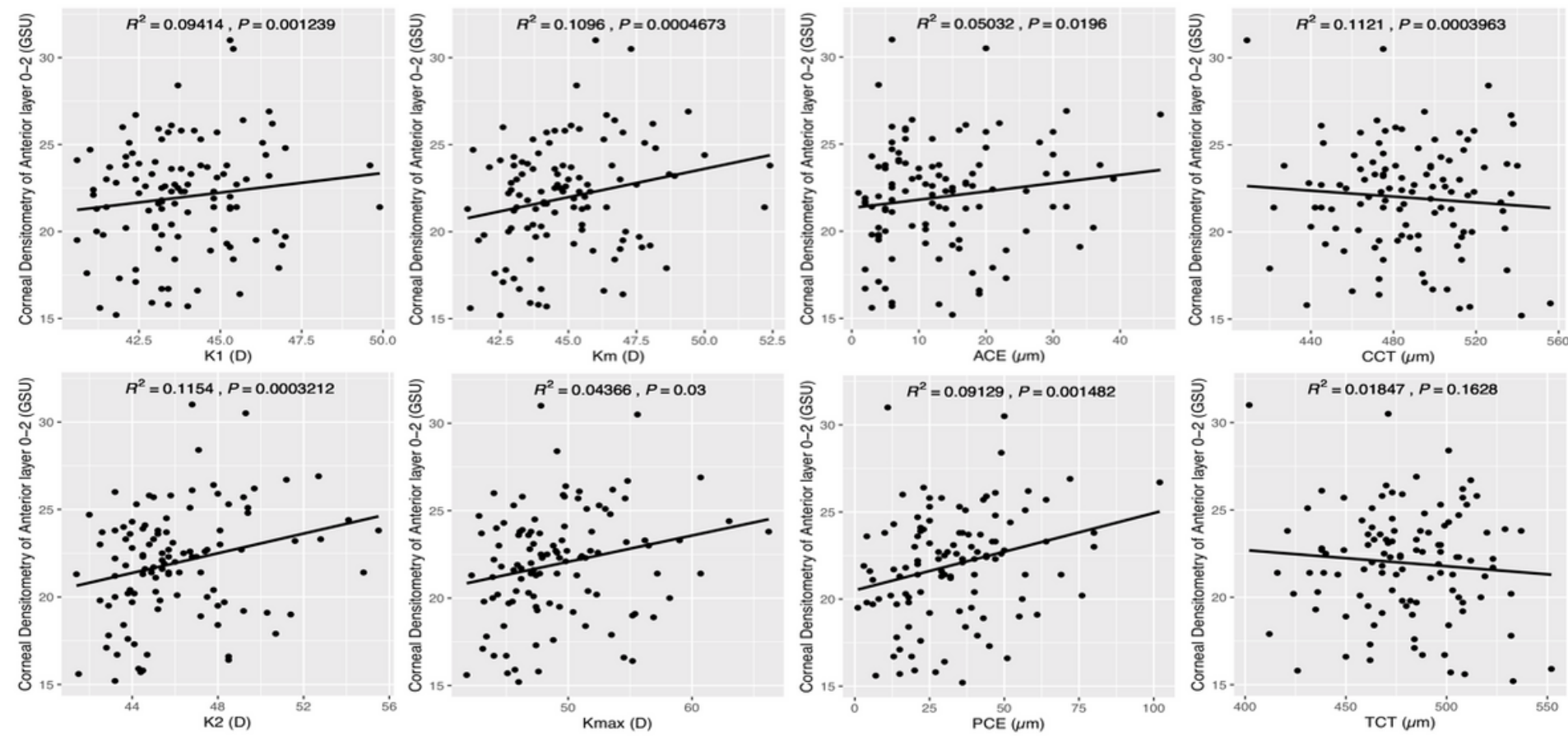

Figure 1

The correlations between corneal densitometry and corneal morphological parameters $\mathrm{K} 1, \mathrm{~K} 2, \mathrm{Km}$, Kmax, ACE, PCE, CCT obtained in the anterior 0-2mm (K1, $R 2=0.094, P=0.001 ; K 2, R 2=0.115, P=0.000 ; K m, R 2=0.110, P=0.00 ; K \max , R 2=0.044, P=0.03 ; A C E, R 2=0.050, P=0.020 ; P C E, R 2=0.091, P=0.001 ; C C T, R 2=0.112$, $P=0.000$ )
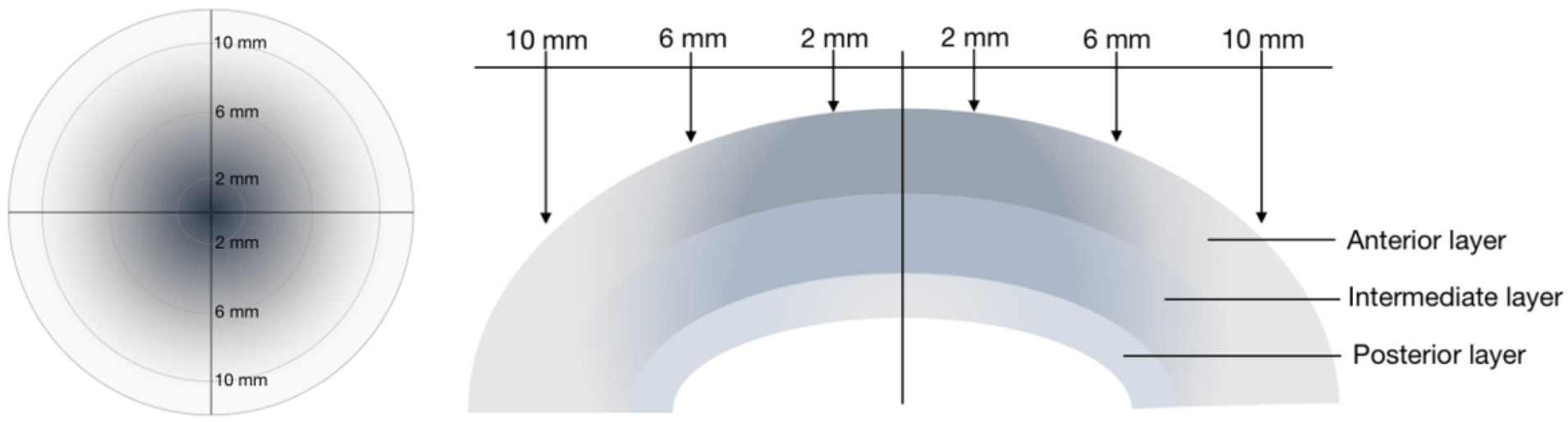

\section{Figure 2}

The densitometry distribution from the anterior to the posterior layers over the annuli of 0 to 2, 2 to 6 and 6 to $10 \mathrm{~mm}$ of a cornea in the KCN eyes with Vogt's striae 